\title{
We need to be (much) better
}

\section{J. H. van Krieken ${ }^{1}$}

Published online: 10 February 2016

(C) Springer-Verlag Berlin Heidelberg 2016

It is now well recognised that optimal care for lymphoma patients starts with an optimal diagnosis. Tools to get to a correct diagnosis are the regularly updated WHO classification, teaching and workshops from (among others) the European Association of Haematopathology and the Society of Hematopathology and the increasing amount of books and articles on the pathology of lymphoma. This enormous amount of knowledge is being used by haematopathologists all over the world and they increasingly rely on techniques like immunohistochemistry and molecular biology.

Many of them share their cases with colleagues within their laboratory or in panels to increase knowledge and quality. Most colleagues therefore will have the same experience as me: there is an enormous variation in the colours of our slides. Since we adapt to the types of artefacts, we introduce in our laboratories this is generally not a big problem. Even for immunohistochemistry that is used for detecting specific expression patterns in certain lymphoma types, this is a small issue: intensity of staining is generally not so relevant.

This situation is now changing. There are increasingly features that we need to assess in tissue samples for selecting targeted therapy. Recently, I attended an interactive meeting on the determination of CD30 expression for selecting patients for anti-CD30 therapy, and I was shocked. I knew how difficult it is to estimate the amount and intensity of staining, but nevertheless, the discordance between experienced haematopathologists was even larger than I had anticipated.
First, there was an issue of staining itself. Staining slides from the same tissue blocks in different laboratories revealed huge differences, even between laboratories that used the same automated stainer and associated antibodies, protocol and software. Secondly, when we were shown slides and were asked to score using our smartphones, we saw that the same slide was scored by some as having about $10 \%$ positive neoplastic cells and by others about $80 \%$ (we had already discussed the importance of correct terminology: a tumour consists of neoplastic cells and reactive cells of the environment; therefore, the term tumour cells is not precise enough: neoplastic cells are the ones that count!). When the same slides were shown after some teaching, our scores diverged even more....

Therefore, it is crucial that we become much better. The human eye, even that of a pathologist, is not good in quantification of colour intensity and amount. To me, it is obvious that such use of immunohistochemistry can only become reliable, when the staining procedures become way more standardised and the evaluation automated, i.e. using computerised approaches. For years, we know that immunohistochemistry as is used today is not optimal, but usable enough for routine diagnostic practice. Nowadays, this is not sufficient anymore: we need to invest in optimising our techniques and interpretation of the results - a challenge, but necessary for our patients!

J. H. van Krieken

Han.vanKrieken@radboudumc.nl

1 Department of Pathology, Radboud University Medical Centre, P.O. Box 9101, 6500 HB Nijmegen, The Netherlands 